\section{Malformation einer Bronchialarterie mit Shunt in das Pulmonalgefäßbett - seltene Ursache der massiven Hämoptyse}

\author{
M. Wolf ${ }^{1}$, U. Schmidt ${ }^{2}$, R. Bieselt ${ }^{1}$ \\ ${ }^{1}$ Chirurgische Klinik II - Thoraxchirurgie - \\ (Chefarzt: PD Dr. R. Bieselt), Clemenshospital GmbH, \\ Akademisches Lehrkrankenhaus der Westfälischen \\ Wilhelms-Universität, Münster \\ 2 Institut für Pathologie am Clemenshospital, Münster
}

Zusammenfassung: Wir präsentieren den Fall einer ansonsten gesunden 41-jährigen Patientin, die mit akut aufgetretener Expektoration hellroten Blutes aufgenommen wurde. Bei unauffälliger Übersichtsaufnahme der Thoraxorgane zeigte sich fiberbronchoskopisch eine Sickerblutung aus dem Ostium des apikoposterioren Segmentbronchus des linken Lungenoberlappens, die während der Untersuchung exazerbierte. Eine einstweilige Blutstillung konnte durch die Blockade des Oberlappenbronchus mittels eines Doppellumentubus herbeigeführt werden. Die endgültige Sanierung der Blutungsquelle erfolgte nach der Verlegung in unsere Klinik durch eine obere Segmentresektion des linken Lungenoberlappens. Die feingewebliche Aufarbeitung ergab eine im Bereich des apikoposterioren Segmentbronchus gelegene Malformation der Bronchialarterie mit abnormer Gefäßkommunikation zu einem Pulmonalarterienast. Während arteriovenöse Malformationen der Lunge mit pulmonalarteriellem Zufluss in weit über 500 Fällen beschrieben wurden und überwiegend bei Patienten mit hereditärer hämorrhagischer Teleangiektasie (HHT, Morbus Rendu-Osler-Weber) auftreten, ergibt sich aus den 23 mitgeteilten Fällen bronchialarteriell gespeister Malformationen mit Shunt in das Pulmonalgefäßbett kein Hinweis auf Vererblichkeit. Da sich bronchialarteriell und pulmonalarteriell gespeiste Malformationen der Lunge darüber hinaus auch bezüglich ihrer klinischen Symptomatik und Lokalisation in charakteristischer Weise voneinander unterscheiden, sollte eine klare begriffliche Trennung zwischen diesen beiden vorgenommen werden.

Malformation of a Bronchial Artery with Shunt into the Pulmonary Vascular Bed - A Rare Cause of Massive Haemoptysis: We present the case of an otherwise healthy 41-yearold woman, who was admitted after having expectorated bright red blood. While the chest roentgenogram was found to be normal, fiberoptic bronchoscopy revealed mild active bleeding originating from the apicoposterior segmental bronchus of the left upper lobe, which exacerbated during the examination. The bleeding was contained through blockade of the upper lobe bronchus by means of a double-lumen endotracheal tube. After the patient had been referred to our department, we performed a resection of the superior segmental group of the left upper lobe. The histopathological examination of the specimen revealed a malformation of the bronchial artery with an abnormal communication to a branch of the pulmonary artery. The

Pneumologie 2001; 55: 520-526

(c) Georg Thieme Verlag Stuttgart · New York ISSN 0934-8387 malformation was located in close proximity to the apicoposterior segmental bronchus. Whereas arteriovenous malformations with a pulmonary artery branch as feeding vessel have been described in more than 500 cases and primarily affect patients with hereditary hemorrhagic teleangiectasia (HHT, Rendu-Osler-Weber disease), the 23 reported cases of malformations supplied by a branch of the bronchial artery show no evidence of heredity. Furthermore, clinical appearence and localisation show characteristical differences between arteriovenous malformations with pulmonary arterial supply and malformations arising from a bronchial arterial source. Therefore, it seems appropriate to make a clear-cut distinction between these two patterns of vascular malformation of the lung.

\section{Einleitung}

Massive Hämoptysen entstehen meist durch die Ruptur kleiner Gefäße im Bereich abnormer Kurzschlussverbindungen zwischen Bronchialarterien und Pulmonalvenen. Solche aufgrund ihres Zuflusses systemischen Blutdruckverhältnissen ausgesetzte Gefäßverbindungen bilden sich reaktiv im Rahmen der Gefäßproliferation und -erweiterung bei entzündlichen und neoplastischen Erkrankungen der Lunge [1]. Massive Hämoptysen werden zumeist bei diesen beiden Entitäten beobachtet [2,3]. Anlagebedingte abnorme arteriovenöse Kurzschlussverbindungen (arteriovenöse Malformationen, AVM) sind in ihrer überwiegenden Mehrzahl zwischen Pulmonalarterien und -venen, also innerhalb des pulmonalen Niederdrucksystems, ausgebildet, so dass es unter diesen Bedingungen nur sehr selten zu bedrohlichen Hämoptysen kommt [4]. Hingegen verursachen Malformationen mit bronchialarteriellem Zufluss und Shunt in das Pulmonalgefäßbett - analog zu den o.g. reaktiv entstandenen Kurzschlussverbindungen - massive Blutungen in den Bronchialbaum.

\section{Kasuistik}

Aus vollem Wohlbefinden empfand eine 41-jährige Angestellte, Raucherin seit 20 Jahren, während ihrer Mittagspause einen Hustenreiz „wie beim Verschlucken“. Im Hustenanfall expektorierte sie „mehrere Taschentücher voll“ hellroten Blutes. Auf dem Weg zum niedergelassenen Pneumologen folgten wiederholt kurze, jeweils mit hellrot-blutigem Auswurf einhergehende Hustenanfälle. Die Röntgen-Übersichtsaufnahme der Thoraxorgane zeigte einen regelrecht altersentsprechenden Befund (s. Abb.1). Der konsiliarisch in einem auswärtigen Haus tätige Kollege begleitete die Patientin in die 


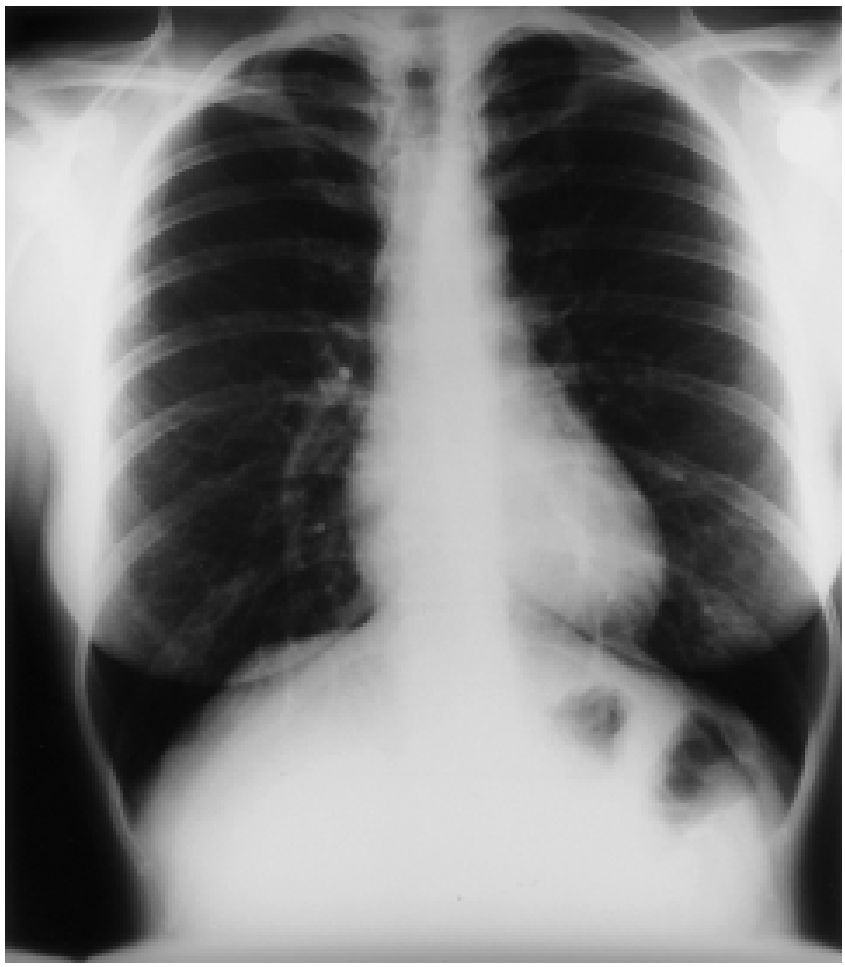

Abb.1 Röntgen-Übersichtsaufnahme nach Expektoration hellroten Blutes: altersentsprechender Befund (p.a.-Strahlengang).

Klinik und führte dort in Anästhesiebereitschaft eine flexible Bronchoskopie durch. Es zeigte sich eine geringe blutige Belegung der Trachea sowie - bei ansonsten unauffälligem Bronchialbaum - eine Sickerblutung aus dem apikoposterioren Segmentbronchus des linken Lungenoberlappens, die bei der Aspiration zur Inspektion deutlich zunahm. Da sich die Blutung weder durch die lokale Instillation von Norepinephrin noch durch den Versuch einer Blockade mit dem Bronchoskop stillen ließ, wurde ein Bronchusblocker in den distalen linken Hauptbronchus platziert. Nach der Deblockade 40 min später kam es erneut zu einer zunehmend starken Blutung, woraufhin die Patientin mit einem Doppellumentubus unter Blockade des linken Oberlappenbronchus intubiert wurde. Die insgesamt während der Untersuchung abgesaugte Blutmenge betrug $300 \mathrm{ml}$. Hb- und kreislaufstabil wurde die Patientin auf die Intensivstation verlegt, die anschließende Röntgenkontrolle zeigte neben einer korrekten Tubuslage nunmehr apikal betonte Transparenzminderungen links (s. Abb. 2). Bei der planmäßigen Extubation 12 Stunden später konnte mittels eines über das linksläufige Tubuslumen eingebrachten Choledoskopes beim Zurückziehen des Tubus eine erneut einsetzende Blutung ausgeschlossen werden. Das anschließend durchgeführte Computertomogramm der Thoraxorgane zeigte bei fehlendem Hinweis auf das Vorliegen einer Tumorformation blutungsbedingte Infiltrationen mit segmentalem Verteilungsmuster (Segmente 1-3) im linken Oberlappen (s. Abb. 3).

Die Patientin wurde mit der Diagnose einer Lungenhämorrhagie unklarer Ursache in unsere Thoraxchirurgische Klinik verlegt, am Folgetag führten wir die linksseitige Thorakotomie über einen posterolateralen Zugang durch. Inspektorisch

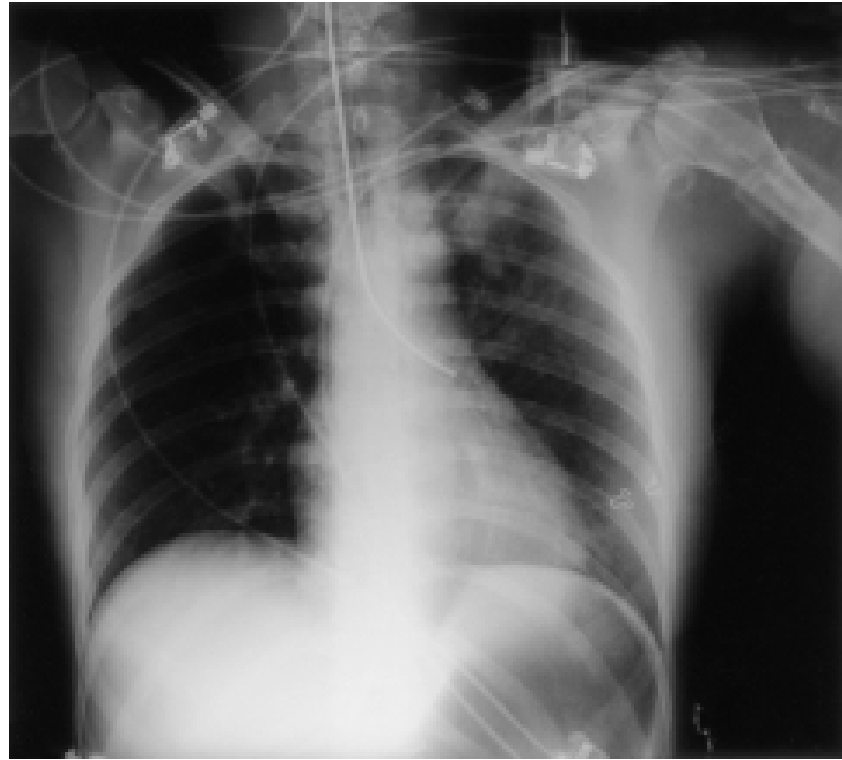

Abb. 2 Röntgen-Übersichtsaufnahme nach Intubation mit einem linksläufigen Doppellumentubus und Blockade des Oberlappenbronchus zur Blutstillung: apikal betonte Transparenzminderung links (Liegendaufnahme a.p.).

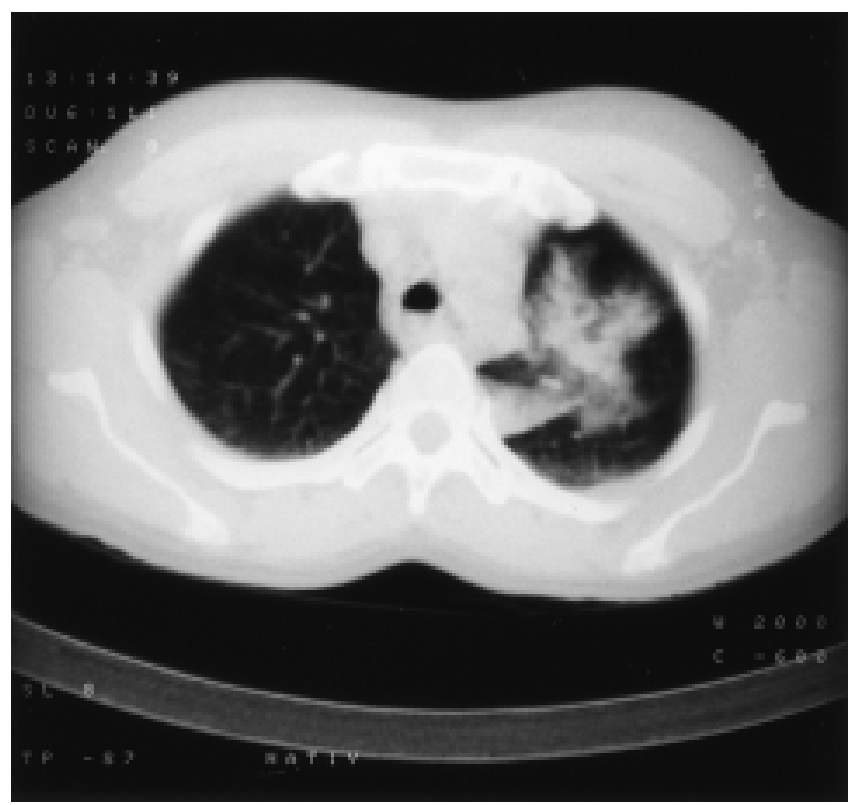

Abb.3 CT des Thorax nach Entfernung des Doppellumentubus: flächige Infiltrationen mit segmentalem Muster in der superioren Segmentgruppe des linken Oberlappens, paravertebrale Atelektase.

zeigte sich auf der Lungenoberfläche eine hauptsächlich die obere Segmentgruppe des Oberlappens betreffende feindisperse Hämatomverfärbung, nebenbefundlich lag eine mäßiggradige Anthrakose vor. Die Palpation ergab keinen Anhaltspunkt für eine Tumorbildung oder suspekte Lymphknotenvergrößerungen. Wir führten eine anatomische Resektion der superioren Segmentgruppe des Oberlappens durch. Der postoperative Verlauf gestaltete sich glatt und komplikationslos. Bei einer Nachbeobachtungszeit von mittlerweile einem 
halben Jahr ist es zu keinem erneuten Blutungsereignis gekommen.

Bei der feingeweblichen Aufarbeitung des Operationspräparates zeigten sich im Bereich eines Segmentbronchus $-1,5 \mathrm{~cm}$ vom Absetzungsrand entfernt - in parabronchialer Lokalisation vermehrt Anschnitte weitlumiger Gefäße in enger Nachbarschaft zueinander. Eine Kurzschlussverbindung zwischen einem Bronchialarterien- und einem Pulmonalarterienast war nachweisbar; der hierbei beteiligte Bronchialarterienast wies einen pathologischen Wandaufbau mit fokalen Ausdünnungen der Gefäßwandung auf. Insgesamt entsprach der Befund demjenigen einer Malformation der Bronchialarterie mit arterioarteriellem Shunt in das Pulmonalgefäßbett (s. Abb. 4,5,6).

\section{Diskussion}

Bluthusten wird von betroffenen Patienten subjektiv als ausgeprochen bedrohliches Ereignis wahrgenommen. Da der tracheobronchiale Totraum des Erwachsenen ein Volumen von $100-200 \mathrm{ml}$ aufweist, gilt eine Blutung von $200 \mathrm{ml} / 24 \mathrm{~h}$ oder mehr insofern als potenziell vital gefährdend, als sie zur Asphyxie führen kann. Vielfach wird daher dieser Grenzwert zur Bezeichnung einer massiven Hämoptyse verwendet [5,6]. Dweik und Stoller [7] kommen nach einer Analyse von Veröffentlichungen, die über einen Zeitraum von 30 Jahren bis zum Jahr 1997 publiziert wurden, zu dem Schluss, dass den massiven Hämoptysen ein über den genannten Zeitraum weitgehend konstant dominierendes Spektrum von Erkrankungen zugrunde liegt: Lungentuberkulose, Bronchiektasen, Lungenabszesse sowie Bronchialkarzinome. Wie einleitend erwähnt, bilden sich bei diesen entzündlichen und neoplastischen Erkrankungen reaktiv - vermutlich als Antwort auf angiogene Stimuli - arteriovenöse Gefäßkommunikationen, die von systemischen Zuflüssen, meist Bronchialarterienästen, gespeist werden. Fallweise kann bei schweren entzündlich-einschmelzenden Erkrankungen der Lunge auch ein mykotisches Pseudoaneurysma eines Pulmonalarterienastes der Ausgangspunkt einer lebensbedrohlichen Blutung sein [8].

Zu den seltenen Ursachen massiver Hämoptysen zählen AVM der Lunge. Am häufigsten werden diese innerhalb des pulmonalen Niederdrucksystems vorgefunden. Der erste solche Fall wurde von Churton [9] Ende des 19. Jahrhunderts autoptisch beschrieben. Er betraf einen 12-jährigen Jungen mit multiplen, zwischen Pulmonalarterien und -venenästen ausgebildeten arteriovenösen Aneurysmen. Seit Ende der 40er Jahre ist die Assoziation pulmonalarteriell gespeister AVM mit der HHT bekannt [10]. Bei dieser regional in sehr unterschiedlicher Häufigkeit auftretenden dominant vererbbaren Erkrankung (Inzidenz: 2,5-43:100000 Einwohner) kommt es bereits im Kindesalter zu rezidivierendem Nasenbluten, um das 20. Lebensjahr treten mukokutane Teleangiektasien auf. 15$30 \%$ der betroffenen Patienten weisen AVM auf, wobei - ohne dass hierfür bislang eine Erklärung gefunden werden konnte - Frauen in einem Verhältnis von 1,6:1 überwiegen [11]. Die Patienten werden meist im mittleren Lebensalter durch die Folgen des extrakardialen Rechts-links-Shuntes (Zyanose, Belastungsdyspnoe, Trommelschlegelfinger, Polyglobulie, paradoxe Thrombembolien) symptomatisch. Umgekehrt liegt bei bis $\mathrm{zu} 80 \%$ der im pulmonalen Niederdrucksystem ausge-

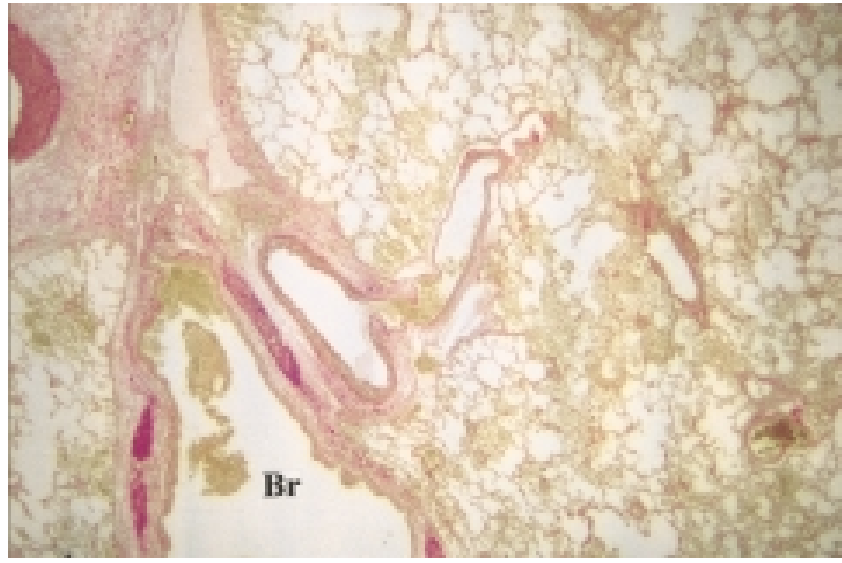

Abb. 4 Übersichtsvergrößerung eines Segmentbronchus ( $\mathrm{Br}$ ) mit parabronchialen Blut- und Lymphgefäßen, zahlreiche eingeblutete Alveolen (Elastika-van-Gieson, 18×).

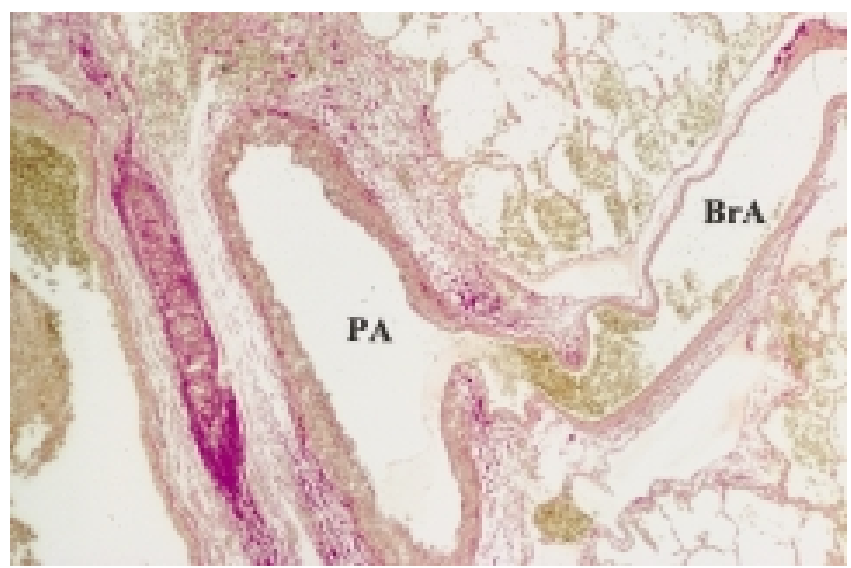

Abb. 5 Stärker vergrößerter Ausschnitt aus Abb. 4: Bronchialarterienast $(\mathrm{BrA})$ mit z. T. erheblicher Verschmälerung seiner Wandung und direktem Übergang in einen Pulmonalarterienast (PA). Im Übergangsbereich fehlt die im Falle physiologischer arterioarterieller Anastomosen (sog. Sperrarterien) typischerweise reichlich ausgebildete Längsmuskelschicht $(46 \times)$.

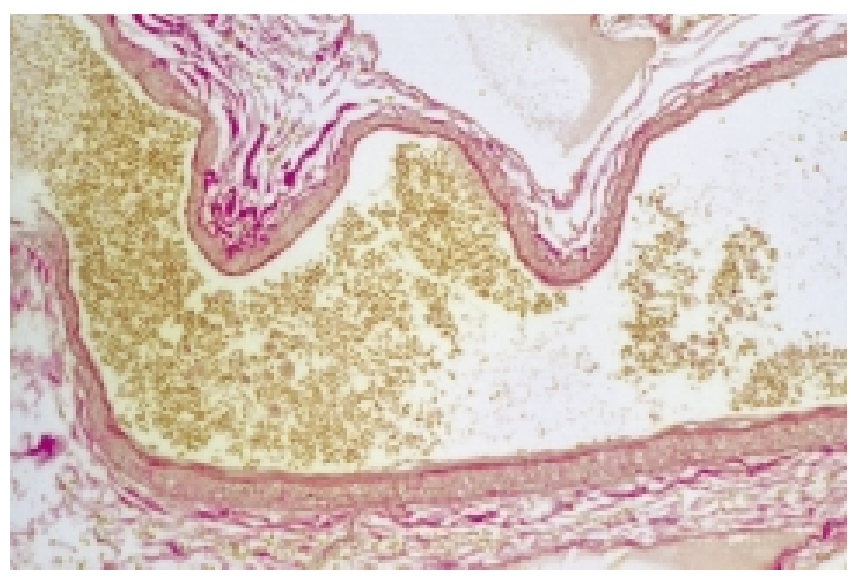

Abb. 6 Stärkere Vergrößerung des in Abb. 5 mit BrA bezeichneten Gefäßes. Die nun deutlich sichtbare Elastika interna und muskuläre Media erlauben seine sichere Identifizierung als Bronchialarterienast $(185 \times)$ 
bildeten AVM eine HHT vor [1]. Über 500 Fälle wurden unter einer Vielzahl von Bezeichnungen (arteriovenöse Lungenfistel, arteriovenöses Angiom, Hämangiom, Hamartom, arteriovenöses Aneurysma) veröffentlicht, bis Burke 1986 [12,13] die einheitliche Verwendung des Begriffes pulmonale arteriovenöse Malformation (PAVM) vorschlug. Lokalisiert sind die überwiegend multipel und bilateral auftretenden PAVM meist in den Lungenunterlappen, häufiger als eine zentrale Lage wird eine subpleurale beschrieben $[4,14,15]$. In einer Serie von 143 Patienten mit HHT und PAVM waren Blutungskomplikationen bei insgesamt 11 Patienten zu konstatieren, bei 5 Patienten kam es zu relevanten Hämoptysen, bei weiteren 5 zu spontanen Hämatothoraces, bei einer Patientin traten sukzessive beide Komplikationen auf [16].

Zu bronchialarteriell gespeisten Malformationen mit Shunt in das Pulmonalgefäßbett gibt es nur wenige Mitteilungen. Aus funktioneller Sicht liegt bei ihnen ein extrakardialer Linksrechts-Shunt (bei Kommunikation der Bronchialarterie mit einer Pulmonalarterie) oder ein Links-links-Shunt (bei Kommunikation der Bronchialarterie mit einer Pulmonalvene) vor. v. Babo u. Mitarb. [17] berichteten 1976 über drei Patienten (24, 29, $42 \mathrm{~J}$.), bei denen es ohne Prodromi zu rezidivierenden Hämoptysen hellroten Blutes gekommen war. Bei bronchoskopisch nicht sicher lokalisierbarer Blutungsquelle wurde eine selektive Angiographie der Bronchialarterie durchgeführt, die jeweils um Segmentbronchien angeordnete Gefäßnetze mit bronchialarteriellem Zufluss und Shuntbildung darstellte. Bei den beiden jüngeren Patienten wurde eine Lappenresektion durchgeführt, der ältere Patient verweigerte jede Therapie. Die feingeweblichen Befunde der beiden operierten Patienten wurden später von Cain und Spanel [18] publiziert, die gleichartige Veränderungen von Bronchialarterienästen in weiteren drei Operationspräparaten gesehen hatten (detaillierte klinische Angaben zu diesen Patienten sind in der histomorphologisch ausgerichteten Arbeit nicht enthalten). Sie bewerteten das in diesen insgesamt 5 Fällen jeweils in der Wand von Segmentbronchien sektorförmig vorgefundene Gefäßkonvolut als angeborene Fehlbildung der Bronchialarterie und bezeichneten es als Angioma arteriovenosum racemosum.

Den letzten in der Literatur nachweisbaren histologisch dokumentierten Fall teilen Sukoh u. Mitarb. [19] 1999 mit. Als primäres racemöses Hämangiom bezeichnen die Autoren die Veränderungen im Bereich des Lappenbronchus und dessen Segmentaufzweigung im Mittellappenresektat einer 38-jährigen Patientin. Die Frau war aufgrund einer aus voller Gesundheit aufgetretenen massiven Hämoptyse aufgenommen worden, als deren Ausgangspunkt im blutungsfreien Intervall eine kleine pulsatile Protrusion im Mittellappenbronchus identifiziert werden konnte, die unter der bronchoskopischen Untersuchung erneut massiv blutete.

Eine Zusammenstellung aller bislang veröffentlichten Fälle von Malformationen der Bronchialarterie mit Shunt in das Pulmonalgefäßbett ist in Tab.1 aufgeführt. Aus ihr geht hervor, dass die Geschlechterverteilung gleichwichtig ist und sich Fälle in allen Altersgruppen finden, vorwiegend jedoch jüngere Erwachsene betroffen sind. Darüber hinaus ist erkennbar, dass das klinische Bild uniform durch das Auftreten von meist massiven Hämoptysen bestimmt wurde und die Malformation überwiegend im Bereich von Segmentbron- chien lokalisiert war. Was die Art der Therapie angeht, so ist festzustellen, dass zumeist Resektionen unterschiedlichen Ausmaßes vorgenommen wurden. In einem Fall wurde zusätzlich die Arteria bronchialis ligiert, in einem weiteren ausschließlich eine thorakoskopische Ligatur mit Durchtrennung der Arteria bronchialis durchgeführt. Bei der jüngsten Patientin sowie den beiden ältesten Patienten wurde die Malformation jeweils selektiv embolisiert.

Stellt man PAVM und Malformationen der Bronchialarterie mit Shunt in das Pulmonalgefäßbett hinsichtlich einiger wesentlicher Charakteristika einander gegenüber, so ergibt sich ein deutlich differierendes Bild (s. Tab. 2).

Was die Nomenklatur angeht, so erscheint eine Vereinheitlichung der Begriffe wie sie im Hinblick auf die arteriovenösen Gefäßkommunikationen im pulmonalen Niederdrucksystem mit der Bezeichnung PAVM gelungen ist, wünschenswert. Da die Bezeichnung Malformation den vermuteten Fehlbildungscharakter hervorhebt ist sie auch bei den hier behandelten Veränderungen von Bronchialarterienästen anwendbar und mithin den Benennungen Angiom, Arteriom oder Hämangiom vorzuziehen. Eine begriffliche Unterscheidung von den „konventionellen“ PAVM forderten Pouwels u. Mitarb. [22] 1992 und schlugen den Terminus S-PAVM (systemic to pulmonary arteriovenous malformation) vor. Die Autoren subsumieren unter diesen Begriff jedoch auch die Vielzahl von Fällen aberrierender systemischer Zuflüsse (etwa aus atypischen Ästen der thorakalen Aorta, Arterien der Brustwand oder Koronararterien) zu abnormen Gefäßkommunikationen der Lunge, die ganz überwiegend posttraumatischer, postoperativer oder postentzündlicher Genese sind [26-28] und somit unseres Erachtens gerade nicht mit der Bezeichnung Malformation belegt werden sollten. Für die hier behandelten orthotopen, überwiegend im Bereich der Segmentbronchien lokalisierten Bronchialarterienäste erscheint die von Soda u. Mitarb. [23] 1995 verwendete Bezeichnung AVM der Bronchialarterie (bronchial AVM) genauer. Wie die in ihrer Arbeit präsentierte Bronchialarteriographie allerdings zeigt, erfolgte der Kontrastmittelabfluss über einen Pulmonalarterienast, so dass hier offenbar ein arterioarterieller und kein arteriovenöser Shunt vorlag. Das gleiche Dilemma ist auch bei den zwei operierten Fällen in der Arbeit von v. Babo u. Mitarb. [17] erkennbar: Obschon es in beiden Fällen bronchialarteriographisch zu einer Frühkontrastierung von Pulmonalarterienästen kam lautete die histologische Diagnose Angioma arteriovenosum racemosum [18]. Als traubenartig (razemös) gestaltet stuften Pathologen des 19. Jahrhunderts vor allem im zentralen Nervensystem vorgefundene Gefäßgeschwülste ein, die sie daher Angioma arteriale racemosum oder Rankenangiom nannten [29]. In diesen beschrieb Jores 1924 „das Vorkommen eines direkten Überganges arterieller Gefäße in venöse unter Fortfall der Kapillaren“, also abnorme arteriovenöse Gefäßkommunikationen [30]. Wie die Gewebeschnitte unseres Falles zeigen, kann es sich bei Malformationen der Bronchialarterie um durchaus diskrete Läsionen handeln. Bei den von anderen Autoren vorgestellten Bronchialarteriographien sieht man überwiegend feine Gefäßnetze und keine größeren, traubenartigen Gefäßkonvolute. Hinsichtlich ihrer Größe scheint bei Malformationen der Bronchialarterie ein ähnlich weites Spektrum zu bestehen wie bei den PAVM. 
Tab. 1 In der Literatur mitgeteilte Fälle von Malformationen der Bronchialarterie mit Shunt in das Pulmonalgefäßbett

\begin{tabular}{|c|c|c|c|c|c|c|}
\hline Autor & Jahr & $\mathrm{n}$ & $\begin{array}{l}\text { Alter/ } \\
\text { Geschlecht }\end{array}$ & Symptome & Lokalisation & Therapie \\
\hline v.Babo [17] & 1976 & 3 & $\begin{array}{l}24 \mathrm{w} \\
29 \mathrm{~m} \\
42 \mathrm{~m}\end{array}$ & $\begin{array}{l}\text { Hämoptyse } \\
\text { Hämoptyse } \\
\text { blutiges Sputum }\end{array}$ & $\begin{array}{l}2 \text { Segmentbronchien (re Unterlappen) } \\
\text { Segmentbronchus } \mathrm{B}^{4} \mathrm{li} \\
\text { Mittellappenbronchus re }\end{array}$ & $\begin{array}{l}\text { Lobektomie } \\
\text { Lobektomie } \\
\text { keine }\end{array}$ \\
\hline Cain [18] & 1980 & 3 & $\begin{array}{l}\text { k. A. } \\
\text { k.A. } \\
\text { k.A. }\end{array}$ & $\begin{array}{l}\text { Hämoptyse } \\
\text { Hämoptyse } \\
\text { Hämoptyse }\end{array}$ & $\begin{array}{l}\text { Segmentbronchus } \\
\text { Segmentbronchus } \\
\text { Segmentbronchus }\end{array}$ & $\begin{array}{l}\text { operativ } \\
\text { operativ } \\
\text { operativ }\end{array}$ \\
\hline Tanigawa* & 1981 & 1 & $40 \mathrm{~m}$ & massive Hämoptyse & Segmentbronchus $\mathrm{B}^{9}$ re & Lobektomie \\
\hline Yagi* & 1983 & 1 & $52 \mathrm{w}$ & massive Hämoptyse & Segmentbronchien $B^{8-10}$ re & Lobektomie \\
\hline Mark [20] & 1984 & 1 & k. A. & massive Hämoptyse & k. A. & k. A. \\
\hline Nagai* $^{*}$ & 1987 & 1 & $35 \mathrm{~m}$ & massive Hämoptyse & Segmentbronchien $B^{8-10}$ re & Lobektomie \\
\hline Sheffield [21] & 1988 & 1 & $71 \mathrm{w}$ & massive Hämoptyse & Segmentbronchus $B^{1}$ li & Lobektomie \\
\hline Satio* & 1989 & 1 & $29 w$ & massive Hämoptyse & $\begin{array}{l}\text { Segmentbronchien } B^{4-5} \text { re, } \\
\text { Segmentbronchus } B^{5} \text { li }\end{array}$ & keine \\
\hline Nakagawa* & 1989 & 2 & $\begin{array}{l}72 \mathrm{w} \\
45 \mathrm{~m}\end{array}$ & $\begin{array}{l}\text { massive Hämoptyse } \\
\text { massive Hämoptyse }\end{array}$ & $\begin{array}{l}\text { Segmentbronchus } B^{4} \mathrm{li} \\
\text { Segmentbronchus } B^{3} \mathrm{li}\end{array}$ & $\begin{array}{l}\text { Lobektomie } \\
\text { Segmentresektion }\end{array}$ \\
\hline Suda* & 1990 & 1 & $18 \mathrm{~m}$ & massive Hämoptyse & Segmentbronchien $B^{6-10}$ re & Lobektomie \\
\hline Pouwels [22] & 1992 & 1 & $21 \mathrm{~m}$ & massive Hämoptyse & $\begin{array}{l}\text { Mittel- und Unterlappen- } \\
\text { Bronchus re }\end{array}$ & untere Bilobektomie \\
\hline Oshika* & 1993 & 1 & $74 \mathrm{~m}$ & Hämoptyse & Segmentbronchus $\mathrm{B}^{5}$ re & Embolisation \\
\hline Soda [23] & 1995 & 1 & $52 \mathrm{~m}$ & Hämoptyse & Subsegmentbronchus $\mathrm{B}^{3 \mathrm{a}}$ re & $\begin{array}{l}\text { Segmentresektion, Ligatur } \\
\text { der A. bronchialis }\end{array}$ \\
\hline Shafari [24] & 1996 & 1 & $11 \mathrm{w}$ & massive Hämoptyse & Unterlappenbronchus re & Embolisation \\
\hline Ohshima * & 1996 & 1 & $80 w$ & massive Hämoptyse & Segmentbronchien $\mathrm{B}^{4-5}$ bds & Embolisation \\
\hline Sukoh [19] & 1999 & 1 & $38 w$ & massive Hämoptyse & $\begin{array}{l}\text { Mittellappenbronchus, } \\
\text { Segmentbronchien } \mathrm{B}^{4-5} \text { re }\end{array}$ & Lobektomie \\
\hline Kanamori [25] & 2000 & 1 & $61 \mathrm{w}$ & Hämoptyse & Segmentbronchien $B^{5}, B^{8}$ re & $\begin{array}{l}\text { Thorakoskopische Ligatur } \\
\text { u. Durchtrennung der } \\
\text { A. bronchialis }\end{array}$ \\
\hline Wolf & 2001 & 1 & $41 \mathrm{w}$ & massive Hämoptyse & Segmentbronchien $\mathrm{B}^{1-2}$ li & $\begin{array}{l}\text { obere Segmentresektion } \\
\left(\mathrm{S}^{1-3}\right)\end{array}$ \\
\hline
\end{tabular}

Mit * gekennzeichnete Literaturstellen (japanische Arbeiten) zitiert nach [19, 25]; k. A. = keine Angabe

\begin{tabular}{|c|c|c|}
\hline & PAVM & MBSP \\
\hline mitgeteilte Fälle & $>500$ & 23 \\
\hline $\begin{array}{l}\text { Geschlechterverteilung } \\
\text { (w:m) }\end{array}$ & $\sim 1,6: 1$ & $\sim 1: 1$ \\
\hline hereditärer Hintergrund & bis $80 \%$ mit der HHT assoziert & nicht vorhanden \\
\hline $\begin{array}{l}\text { Art des vorliegenden } \\
\text { Shuntes }\end{array}$ & extrakardial, rechts-links & $\begin{array}{l}\text { extrakardial, links-rechts } \\
\text { oder links-links }\end{array}$ \\
\hline $\begin{array}{l}\text { shuntbedingte klinische } \\
\text { Symptomatik }\end{array}$ & $\begin{array}{l}\text { Zyanose, Belastungsdyspnoe, } \\
\text { Trommelschlegelfinger u.a. }\end{array}$ & keine \\
\hline Lokalisation & $\begin{array}{l}\text { meist subpleural } \\
\text { meist multipel } \\
\text { meist basal }\end{array}$ & $\begin{array}{l}\text { zentral (meist Segment- } \\
\text { bronchien) } \\
\text { meist solitär } \\
\text { keine augenfällige } \\
\text { Bevorzugung }\end{array}$ \\
\hline $\begin{array}{l}\text { Blutungskomplikationen } \\
\text { (massive Blutung) }\end{array}$ & selten & häufig \\
\hline- Art & $\begin{array}{l}\text { Hämoptyse oder Hämatothorax } \\
(\sim 1: 1)\end{array}$ & Hämoptyse \\
\hline - Farbe & dunkelrot & hellrot \\
\hline
\end{tabular}

Tab. 2 Charakteristika von PAVM und Malformationen der Bronchialarterie mit Shunt in das Pulmonalgefäßbett (MBSP) im Vergleich 
Offenbar unabhängig davon, zu welchem Schenkel des pulmonalen Niederdrucksystems (pulmonalvenöse oder -arterielle Strombahn) abnorme Gefäßkommunikationen ausgebildet waren, präsentierten sich die allermeisten Fälle mit dramatischer Klinik. Die von uns gewählte Bezeichnung Malformation der Bronchialarterie mit Shunt in das Pulmonalgefäßbett umfasst beide Shuntformen. Ungeachtet dieser Überlegungen zur Nomenklatur kommt im klinischen Einzelfall der Frage, ob ein arterioarterieller oder arteriovenöser Shunt vorliegt, vor allem dann Bedeutung zu, wenn therapeutisch die Embolisation der Malformation vorgesehen ist, dies besonders im Hinblick auf die Möglichkeit eines Übertritts des Embolisates in den Systemkreislauf.

\section{Schlussfolgerung}

Wie der präsentierte Fall zeigt, sollte das Vorliegen einer Malformation der Bronchialarterie mit Shunt in das Pulmonalgefäßbett als seltene Ursache der Hämoptyse unter folgenden Umständen erwogen werden:

1. Wenn es sich um eine einmalig oder rezidivierend auftretende heftige Expektoration hellroten Blutes handelt und zugleich

2. das Vorliegen einer der häufigen Ursachen der massiven Hämoptyse (entzündliche und neoplastische Erkrankungen der Lunge) anamnestisch, klinisch, labordiagnostisch und radiologisch mit zureichender Sicherheit ausgeschlossen werden kann.

Als auffallend bezeichneten schon v. Babo u. Mitarb. [17] „die Diskrepanz zwischen der Schwere der Symptomatik und den uncharakteristischen oder sogar unauffälligen Röntgenbefunden der Lunge“. Gelingt es in einem solchen Kontext, den Ursprung der Blutung bronchoskopisch zweifelsfrei zu identifizieren, so ist unseres Erachtens die Indikation zur Operation frühelektiv zu stellen und eine möglichst parenchymsparende Resektion im Gesunden anzustreben. Kann die bronchoskopische Diagnostik die Lage der Blutungsquelle im Bronchialbaum hingegen nicht sicher zuordnen, so muss eine selektive Angiographie der Bronchialarterien vorgenommen werden. Bei anhaltender, durch endobronchiale Maßnahmen nicht kontrollierbarer Blutung oder funktioneller Inoperabilität kann auf diesem Wege zudem eine einstweilige oder definitive Blutstillung durch selektive Embolisation des zuführenden Bronchialarterienastes erreicht werden.

\section{Danksagung}

Wir danken Herrn Dr. med. A. Lingenfelser, niedergelassener Pneumologe in Münster, für die Zuweisung dieses interessanten Falles.

\section{Literatur}

${ }^{1}$ Hughes JM, Luce JM. Pulmonary arteriovenous malformations and other pulmonary vascular abnormalities. In: Murray JF, Nadel JA (Hrsg). Textbook of Respiratory Medicine $3^{\text {rd }}$ ed. Philadelphia: WB Saunders, 2000: 1558 ff.

2 Stoller JK. Diagnosis and management of massive hemoptysis. A review. Respir Care 1992; 37: 564-581
${ }^{3}$ Marshall TJ, Flower CD, Jackson JE. The role of radiology in the investigation and management of patients with haemoptysis. Clin Radiol 1996; 51: $391-400$

${ }^{4}$ Shields TW. Congenital vascular lesions of the lungs. In: Shields TW (Hrsg). General Thoracic Surgery - Vol. 2. Baltimore: Williams \& Wilkins, 1994: 899 ff.

${ }^{5}$ Johnston H, Reisz G. Changing spectrum of hemoptysis. Underlying causes in 148 patients undergoing diagnostic flexible bronchoscopy. Arch Intern Med 1989; 149: 1666-1668

${ }^{6}$ Knott-Craig CJ, Oostuizen G, Rossouw G, Joubert JR, Barnard PM. Management and prognosis of massive hemoptysis: recent experience with 120 patients. J Thorac Cardiovasc Surg 1993; 105: $394-397$

${ }^{7}$ Dweik RA, Stoller JK. Role of bronchoscopy in massive hemoptysis. Clin Chest Med 1999; 20: 89-105

${ }^{8}$ Remy J, Lemaitre L, Lafitte JJ, Vilain MO, Saint Michel J, Steenhouver F. Massive hemoptysis of pulmonary arterial origin: diagnosis and treatment. AJR 1984; 143: 963-969

${ }^{9}$ Churton J. Multiple aneurysm of pulmonary artery. Brit Med J 1897; $1: 1223$

${ }^{10}$ Goldman A. Arteriovenous fistula of the lung: its heredity and clinical aspects. Am Rev Tuberc 1948; 57: 266 - 280

${ }^{11}$ Shovlin CL, Winstock AR, Peters AM, Jackson JE, Hughes JM. Medical complications of pregnancy in hereditary haemorrhagic teleangiectasia. QJ Med 1995; 88: 879-887

${ }^{12}$ Burke CM, Raffin TA. Pulmonary arteriovenous malformations, aneurysms and reflections. Chest 1986; 89: $771-772$

${ }^{13}$ Burke CM, Safai C, Nelson DP, Raffin TA. Pulmonary arteriovenous malformations: a critical update. Am Rev Respir Dis 1986; 134: 334

${ }^{14}$ Dutton JA, Jackson JE, Hughes JM, Whyte MK, Peters AM, Ussov W, Allison DT. Pulmonary arteriovenous malformations: results of treatment with coil embolization in 53 patients. Am J Roentgenol 1995; 165: 1119-1125

${ }^{15}$ White Jr RI, Lynch-Nyhan A, Terry P, Buescher PC, Farmlett EJ, Charnas L, Shuman K, Kim W, Kinnison M, Mitchell SE. Pulmonary arteriovenous malformations: techniques and long-term outcome of embolotherapy. Radiology 1988; 169: 663-669

${ }^{16}$ Ference BA, Shannon TM, White RI, Zavin M, Burdge CM. Lifethreatening pulmonary hemorrhage with pulmonary arteriovenous malformations and hereditary hemorrhagic teleangiectasia. Chest 1994; 106: 1387-1390

${ }^{17}$ v. Babo H, Huzly A, Deininger HK, Barth V. Angiome und angiomartige Veränderungen der Bronchialarterie. Fortschr Röntgenstr 1976; 124: 103 - 110

${ }^{18}$ Cain H, Spanel K. Aetiologie und Morphogenese des sogenannten Bronchusarterioms. Klin Wochenschr 1980; 58: 347-357

${ }^{19}$ Sukoh N, Yamamoto H, Muroya K, Harada T, Suzuki A, Inoue M, Watanabe N, Kuroda R, Kamiizumi H. Primary racemose hemangioma of bronchial artery. Nihon Kokyuki Gakkai Zasshi 1999; 37: $67-71$

${ }^{20}$ Mark EJ. Lung Biopsy Interpretation. Baltimore: Williams \& Wilkins, 1984: 147

${ }^{21}$ Sheffield EA, Moore-Gillon J, Murday AR, Addis BJ. Massive haemoptysis caused by spontaneous rupture of a bronchial artery. Thorax 1988; 43: $71-72$

22 Pouwels HM, Janevski BK, Penn OC, Sie HT, ten Velde GP. Systemic to pulmonary vascular malformation. Eur Respir J 1992; 5: $1288-1291$

${ }^{23}$ Soda H, Oka M, Kohno S, Watanabe M, Takatani H, Hara K. Arteriovenous malformation of the bronchial artery showing endobronchial protrusion. Intern Med 1995; 34: 797-800

${ }^{24}$ Shafari M, Messersmith R, Newman B, Chung Y, Lakier JB. Bronchial arteriovenous malformation in a child with hemoptysis. Angiology 1996; 47: $203-208$ 
${ }^{25}$ Kanamori S, Nakamura H, Nakamoto A, Shinzato T, Tateyama M. Successful thoracoscopic ligation and transection of racemose hemangioma of bronchial artery. Nihon Kokyuki Gakkai Zasshi 2000; 38: $403-407$

${ }^{26}$ Brundage BH, Gomez AC, Cheitlin MD, Gmelich JT. Systemic artery to pulmonary vessel fistulas: report of two cases and a review of the literature. Chest 1972; 62: 19-23

${ }^{27}$ Dines DE, Seward JB, Bernatz PE. Pulmonary arteriovenous fistulas. Mayo Clin Proc 1983; 58: 176-181

${ }^{28}$ Fein AB, Godwin JD, Moore AV, Moran JF, Young WG. Systemic artery-to-pulmonary vascular shunt: a complication of closedtube thoracostomy. AJR 1983; 140: 917 -919

${ }^{29}$ Schück P. Über das Wesen und über die Entstehung des Angioma arteriale racemosum. Berlin: Inaug.-Diss, 1886

${ }^{30}$ Jores L. Arterien. In: Henke, F, Lubarsch O (Hrsg.). Handbuch der Speziellen Pathologischen Anatomie und Histologie - Bd. 2: Herz und Gefäße. Berlin: Springer, 1924: 758-760

\section{Dr. med. M. Wolf}

Chirurgische Klinik II - Thoraxchirurgie Clemenshospital GmbH

Akademisches Lehrkrankenhaus der

Westfälischen Wilhelms-Universität Münster Düesbergweg 124

48153 Münster

E-mail: wolfmi@uni-muenster.de 